\title{
Decisions about cardiopulmonary resuscitation
}

\author{
Time to talk
}

Involving patients in decisions about their care is morally, legally, and clinically desirable, whatever their age. The use of do not resuscitate orders, when a decision is made not to give a patient cardiopulmonary resuscitation, is common. In the United States patient consent is a universal requirement for do not resuscitate orders and is enshrined in hospital policies and state law. In Britain this approach is resisted on the grounds that it replaces the humane tradition of medical care with a formulaic adherence to policy. ${ }^{1}$ But the ethical principle of respect for patients' autonomy and the practical objective of benefiting patients with minimal harm necessitate involving patients in these decisions. ${ }^{2}$

We need to establish humane, valid, and reliable ways of ascertaining patients' views. Only when discussing do not resuscitate orders would harm patients, or when cardiopulmonary resuscitation would be futile, should competent patients not be consulted. Three papers in this week's journal concern the withholding of cardiopulmonary resuscitation: Hill et al and Morgan et al have surveyed doctors' and patients' views on cardiopulmonary resuscitation ( $p$ 1677, ${ }^{3}$ p 1677 ); Doyal and Wilsher discuss the moral and legal implications of do not resuscitate orders for incompetent patients, particularly elderly patients with dementia or stroke (p 16895).

Hill et al and Morgan et al show that agreement is poor between doctors and their patients regarding resuscitation. Hill et al found that most doctors do not believe that patients should be involved in decisions about resuscitation and do not discuss cardiopulmonary resuscitation status with their patients. This survey was of a small sample and had a poor response rate, but the results are borne out elsewhere. ${ }^{6}$

In contrast most patients want to discuss cardiopulmonary resuscitation: these studies found that nearly all patients thought that doctors should discuss plans about cardiopulmonary resuscitation with them. ${ }^{34}$ Interestingly, fewer patients actually want to be involved in making the final decision. ${ }^{37}$ This is a subtle distinction that should be explored to establish the most humane way of discussing cardiopulmonary resuscitation with patients. If discussion of do not resuscitate orders is harmful then deciding not to involve patients is justified. But the evidence for harm would have to be better than that currently available: one American series of five patients who became distressed after discussion of decisions not to resuscitate. ${ }^{8}$

Do patients want cardiopulmonary resuscitation? Both Hill $e t$ al and Morgan et al found that patients are less likely than their doctors to want it. Yet in one group of 67 elderly patients, of whom 60 had been designated not for resuscitation, Liddle et al found that 49 would want it. ${ }^{7}$ The discrepancy between doctors' and patients' views might partly be accounted for by the tendency of elderly patients to overestimate the success rate of cardiopulmonary resuscitation. ${ }^{9}$ Murphy et al showed that when elderly American patients were informed of the true success rate the number choosing cardiopulmonary resuscitation halved. ${ }^{10}$ To make valid choices patients must be properly informed.

How can doctors ascertain their patients' views? The most obvious way is to ask them. Doyal and Wilsher suggest that explicit discussion of decisions not to resuscitate are unnecessary with every competent patient if patients' views can be inferred from other discussions about the goals of treatment. ${ }^{5}$ Whether the views inferred in this way are valid and reliable reflections of patients' choices about resuscitation remains to be shown. The views of chronically ill patients may change over time: in one group of patients with motor neurone disease choices changed over six months. ${ }^{1}$ Patients' views need regular review.

When cardiopulmonary resuscitation is considered to be futile doctors need not discuss or offer it. Broad consensus exists on some of the categories of patients for whom resuscitation has such a poor outcome as to be futile. Patients with advanced terminal cancer and those in whom death is expected in a matter of weeks fall into these categories. ${ }^{12}$ Observational studies have shown poor survival among elderly patients after cardiopulmonary resuscitation (less than $4 \%$ ) in some settings but much better outcomes in others. ${ }^{13}$ Old age itself does not mean that cardiopulmonary resuscitation is futile. Interestingly, Hill et al found that $29 \%$ of the senior doctors in their sample would not resuscitate healthy patients over 70, apparently solely on the basis of age. Conversely, one third of doctors stated that they would resuscitate patients with incurable malignancy. At the very least this suggests some disagreement over the conditions for which cardiopulmonary resuscitation is futile. Further work is needed both to establish prognostic indicators for cardiopulmonary resuscitation and to disseminate this information to doctors.

When patients are incompetent doctors must make choices about resuscitation in their best interests. Doyal and Wilsher state that patients should be considered to be competent unless they are specifically unable to understand the issues and make a rational choice about cardiopulmonary resuscitation. Evidence of incompetence on other issues does not imply incompetence to make choices about resuscitation. Patients with mild or moderate dementia are not automatically incompetent.

Doyal and Wilsher suggest that cardiopulmonary resuscitation would not be legally or morally in the best interests of patients with severe dementia or stroke who have cognitive impairment equivalent to acute severe brain damage. For incompetent patients with less severe dementia cardiopulmonary resuscitation may still be in their best interests unless their ability to flourish as people is compromised to the extent that they cannot make plans or undertake actions.

In practice, establishing competence and determining best interests are complex, and no simple algorithm exists. Decisions about resuscitating incompetent patients must be explicit and reached by consensus among the whole medical team. A written policy is one way of making this more likely. ${ }^{14}$

DOMINIQUE FLORIN Honorary lecturer in public health medicine

Health Promotion Sciences Unit,

Department of Public Health and Policy,

London School of Hygiene and Tropical Medicine, London WC1E 7HT

1 Saunders J. Who's for CPR? I R Coll Physicians Lond 1992;26:254-7.

2 Gillon R. Resuscitation policies-action required. F Med Ethics 1992;18:115-6.

3 Hill ME, McQuillan G, Forsyth M, Heath D. Cardiopulmonary resuscitation-who makes the decision? $B M 7$ 1994:308:1677.

Morgan $R$ King D, Priapati $C$ Rowe J. Views of elderly patients and their relatives on cardiopulmonary resuscitation. BMf 1994;308:1677-8.

5 Doyal $L$, Wilsher $D$. Withholding and withdrawing life sustaining treatment in elderly people: towards formal guidelines. $B M 7$ 1994;308:1689-92.

6 Davies KN, King D, Silas JH. Professional attitudes to cardiopulmonary resuscitation in departments of geriatric and general medicine. $\mathcal{F} R$ Coll Physicians Lond 1993;27:127-30.

7 Liddle J, Gilleard C, Neil A. Elderly patients' and their relatives' views on CPR. Lancet 1993;342:1055. 
8 Schade SG, Muslin H. Do not resuscitate decisions: discussions with patients. F Med Ethics 1989;15:186-90.

9 Miller DL, Jahnigen DW, Gorbien MJ, Simbartl L. Cardiopulmonary resuscitation: how useful? Attitudes and knowledge of an elderly population. Arch Intern Med 1992;152:578-82.

10 Murphy DJ, Burrows D, Santilli RN, Kemp AW, Tenner S, Kreling B, et al. The influence of the probability of survival on patients' preferences regarding cardiopulmonary resuscitation. N Engl f Med 1994;330:545-9.
11 Silverstein MD, Stocking CB, Antel JP, Beckwith J, Roos RP, Siegler M. Amyotrophic lateral sclerosis and life-sustaining therapy: patients' desires for information, participation in decision making, and life sustaining therapy. Mayo Clin Pro 1991;66:906-13.

2 Buckman R. Who's for CPR? 9 R Coll Physicians Lond 1992;26:461-2

13 Smith EM, Hastie IR. Resuscitation status of the elderly. $\mathcal{F}$ R Coll Physicians Lond 1992;26:377-9.

14 Florin D. "Do not resuscitate" orders: the need for a policy. $f R$ Coll Physicians Lond 1993;27 $135-8$

\title{
Retaining personal medical records of children who have had chemotherapy and radiotherapy
}

\author{
New guidelines needed
}

Most children who develop cancer can now expect their primary disease to be cured, and this is largely attributable to intensive chemotherapy and radiotherapy. ${ }^{1}$ Long term follow up, however, shows that effects related to treatment may become evident many years later. ${ }^{1-4}$

When children have been exposed to radiotherapy or chemotherapy important clinical reasons exist for maintaining a detailed record of such treatments. The patients may present many years (possibly decades) after treatment and ask about their prospects of being fertile, the chance of an adverse outcome of pregnancy, or the chance of their children developing serious genetic disease. Cardiomyopathy or lung dysfunction related to treatment may be diagnosed. They may also present with a late recurrence of their cancer or with a second primary neoplasm. In all of these circumstances the details of previous exposure to radiotherapy and individual cytotoxic drugs are crucially important to diagnosis and rational and safe planning of further treatment.

Now that two thirds of patients treated for childhood cancer in Britain survive for at least five years an increasingly important question when future treatment protocols are planned concerns the risks of adverse long term outcomes related to treatment. The aim of treatment now is cure at minimum cost in terms of late effects of treatment. Retaining details of such treatment is invaluable for subsequent research. Much useful information relating to the long term effects of radiotherapy and particular cytotoxic drugs may be obtained from retrospectively ascertained cohort and case-control studies, provided that the details of treatment are still available for most patients.

Several such national epidemiological studies have been completed of patients treated for childhood cancer in Britain since 1950 who survived at least the initial few years after diagnosis. Of particular importance are studies of long term survival and detailed investigations of the causes of deaths that occur at least five years after diagnosis. ${ }^{1}$ These provide clarification of the curability of disease, estimates of the risk of deaths related to treatment, and potentially preventable deaths. Also studies of second primary neoplasms ${ }^{23}$ and of the outcomes of pregnancies and the health of offspring of survivors further improve our understanding of the heritability of different neoplasms and identify adverse outcomes associated with particular forms of treatment. ${ }^{46}$

During our studies it has become increasingly apparent that medical notes relating to radiotherapy and chemotherapy are being destroyed prematurely. Sometimes, before destruction, copies of a sample of the medical records have been taken and stored on microfilm or microfiche. But even if such archiving has been carried out it has often been done by clerical staff with little knowledge of which aspects of the records are most important in terms of long term clinical and research needs.
As a result, details of radiotherapy and chemotherapy are sometimes lost while pages of blood counts are retained.

The Department of Health has produced national guidelines on the preservation, retention, and destruction of medical records. ${ }^{78}$ Broadly, records relating to children and young people should be retained until the patient's 25th birthday, or 26th if entry was made when the young person was 17 , or eight years after the patient's death if sooner. Obstetric records should be retained for 25 years, or eight years after the death of the child if sooner.

Policies relating to the retention and destruction of personal NHS medical records vary widely among hospitals. Furthermore, the national guidelines are often not adhered to and notes are being destroyed before the prescribed periods have elapsed.

For clinical purposes detailed records of radiotherapy and chemotherapy are needed for all patients while they are alive. For research purposes records on children who survive at least five years after such treatments should be retained. The minimum requirements for what should be retained are a full copy of the details of radiotherapy, including daily doses and any diagrams of sites irradiated, and at least the cumulative dose of each cytotoxic drug received (or sufficient details to allow calculation of a cumulative dose).

The current national guidelines need urgent review and should specify the minimum amount of information that should be retained for survivors of childhood cancer. In addition, some means of ensuring adherence to the guidelines is necessary. Given the competing demands for resources in the NHS, the need to retain this important information can easily be overlooked. Only 1200 new cases of childhood cancer are diagnosed each year in Britain, and these patients are, of necessity, exposed to extremely toxic treatment. It is essential that any information that might be gleaned from past experience is not lost; in the worst circumstances such losses could result in avoidable deaths.

Although we have concentrated on childhood cancer, similar arguments apply to young adults treated for cancer ${ }^{9-12}$ and to patients given similar treatments for other conditions -for example, transplant recipients. ${ }^{13}$

M M HAWKINS Epidemiologist

Childhood Cancer Research Group,

University of Oxford,

Oxford OX2 6H]

A W CRAFT

Chairman of the United Kingdom Children's Cancer Study Group

Department of Child Health,

University of Newcastle,

Newcastle upon Tyne NE1 7RU

1 Robertson CM, Hawkins MM, Kingston JE. Late deaths and survival after childhood cancer: implications for cure. $B M \mathcal{F}$ (in press). 